\title{
ASTEROSEISMOLOGY FROM THE GROUND
}

\author{
S. FRANDSEN \\ Institute of Physics and Astronomy, Århus University, Denmark
}

ABSTRACT In stars which pulsate in more than one mode, be it acoustic or gravity modes, radial or non-radial, we have a chance to study the interior structure by use of seismic techniques. Generally the greater the number of modes observable the greater the chances will be to obtain valuable direct information about the stellar interior not accessible by any other technique.

The observational problem of acquiring high quality data divides the stars where seismic techniques can be applied into two groups: A group of stars with intensity amplitudes in the range of millimagnitudes and the stars with very small amplitudes around $10 \mu$ magnitudes or less. The first group contains the white dwarfs, the rapidly oscillating Ap stars, the $\delta$-Scuti stars and the $\beta$-Cephei stars. The modes are excited directly or indirectly by some form of $\kappa$-mechanism due to an opacity source which vary from class to class. The second group is made up of stars with outer convective envelopes which generate acoustic noise that may excite resonant modes, often a very large number.

This paper is a discussion of the possibilities for observing these stars with ground based techniques, and also a statement about what can not be done from the ground.

\section{INTRODUCTION}

For a general idea about the subject I recommend the papers by Brown (1990a) and Baade (1991). Also the proceedings from meetings in Aarhus, Tenerife and Cambridge contain articles that are relevant.

The paper is organized in four sections. The first defines the observational arena for asteroseismology. The techniques to be applied depends on the characteristics of the type of stars to be studied. The important parameters are the period $P$, the semi-amplitude $A$, the magnitudes of the objects under study $m_{V}$ and the number of modes in the pulsation spectra. The second section addresses the Doppler techniques and the following the photometric techniques. The final section is a small discussion of the shortcomings of ground based observations and the necessity to observe from space. 


\section{THE MAIN AREAS OF GROUND-BASED ASTEROSEISMOLOGY}

Seismic techniques can be applied to a number of different types of stars. For each category the set of modes define a specific observational and theoretical scenario, which is special and different among the types of objects. Groundbased observations contribute mainly in the following areas, which in some cases represent the full data needed, in others only supplementary data to space observations.

- 1 Spectroscopy and Doppler velocity studies due to the weight and size of the equipment to perform such studies.

- 2 Long term monitoring (stellar cycles/evolutionary changes)

- 3 Faint objects where photon noise dominates scintillation noise, i.e. white dwarfs. This depends obviously on telescope size.

- 4 Future interferometric measurements (differentiel speckle photometry).

- 5 Doppler imaging which demand very high resolution and high $\mathrm{S} / \mathrm{N}$ ratios.

- 6 Simultaneous support observations with space experiments.

What's needed? This question is related to the physics of the objects, but generally the data required are more time consuming and complicated to obtain than for classical variables or for single stars in general. A list of properties of the data could look like

- 1 Very low noise measurements (no $1 / f$ noise for $\beta$ Cephei or $\delta$-Scuti stars)

- 2 Long time series with few interruptions to produce high quality spectra.

- 3 Doppler imaging to help mode identifation.

- 4 Devoted studies of a small set of key objects (compare with the work on the Sun!).

- 5 Development of new, more sensitive instrumentation for extreme precision in Doppler studies. The lowest noise obtained until date is $30 \mathrm{cms}^{-1}$ (Pottasch et al. 1992). The goal is to reach $1 \mathrm{cms}^{-1}$.

In order to meet the above demands new techniques and devoted observations are required. Somebody must invest an effort in organizing networks of modern state of the art instrumentation. Small or medium size telescopes allocated for extended periods to these studies will be needed and to make life acceptable for the observers, they should be highly automized.

The stars that are the main candidates for seismic studies can be put into five classes, which have characteristics as given in Table I.

Solar type stars is yet an area to be explored due to the minute amplitudes of the oscillations. But the white dwarfs observed by the WET consortium (Nather et al. 1990) show oscillation spectra of similar richness representing extremely valuable data for analyzing the interior structure of the white dwarfs. A spectrum is shown as Figure I. The other classes present spectra with fewer 
TABLE I Classes of variables

\begin{tabular}{|l|l|l|l|l|l|}
\hline Class & Period & Amplitude & Magnitudes & $\begin{array}{l}\text { Observ. } \\
\text { modes }\end{array}$ & Occur. \\
\hline$\delta$-Scuti & $0.5-7 \mathrm{~h}$ & $1-50 \mathrm{mmag}$ & $2<m<15$ & $1-5$ & single/clust. \\
$\beta$-Cephei & $3-6 \mathrm{~h}$ & $10-100 \mathrm{mmag}$ & $2<m<15$ & $1-2$ & single/clust. \\
roAp's & $4-15 \mathrm{~m}$ & $10-1000 \mu \mathrm{mag}$ & $4<m<8$ & $1-10$ & single \\
Wh. dw. & $3-40 \mathrm{~m}$ & $1-100 \mathrm{mmag}$ & $13<m<16$ & $1-100$ & single \\
Solar *'s & $3-40 \mathrm{~m}$ & $1-100 \mu \mathrm{mag}$ & $0<m<6$ & $30-50^{a}$ & single \\
\hline
\end{tabular}

${ }^{a}$ Full disk modes only

${ }^{b}$ Assuming velocity measurements only

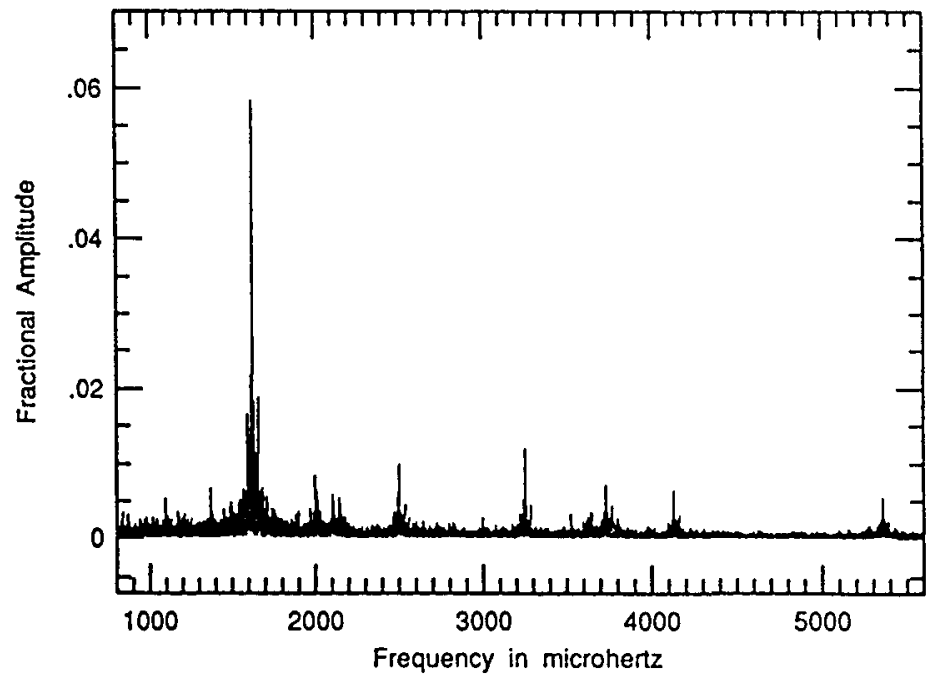

FIGURE I Part of amplitude spectrum of the white dwarf G29-38 from Winget et al. 1990 
modes. Given stars enough this still opens up a very promising field of interpretation. Examples of $\delta$-Scuti type spectra are found in Michel and Baglin (1991), the STEPHI program, and Mantegazza et al. (1992).

\section{VELOCITY STUDIES}

Velocity studies have been carried out by the extension of solar techniques or by modifications of classical spectrographs to obtain the stability needed.

In Table II a list of groups is given that have attempted to observe Doppler velocities due to solar type observations or suggested techniques yet to be tested to do so.

TABLE II Instrumentation based on spectroscopy

\begin{tabular}{|c|c|c|}
\hline Group/Reference & Technique & Usage \\
\hline $\begin{array}{l}\text { 1 Channel/1 line } \\
\text { a. Nice group } \\
\text { Schmider 1988, } 1990 \\
\text { b. Birmingham group } \\
\text { Innis et al. 1990 } \\
\text { c. Imperial College } \\
\text { Reay et al. 1986 } \\
\text { d. Harvey Butcher group } \\
\text { Pottasch et al. } 1992 \\
\end{array}$ & $\begin{array}{l}\mathrm{Na} \text { resonance cell } \\
\mathrm{K} \text { resonance cell } \\
\text { F-P filter } \\
\text { Michelson Interferometer } \\
\text { F-P filter } \\
\text { fiber + scrambler }\end{array}$ & $\begin{array}{l}\alpha \text { Cen, Procyon } \\
\text { Procyon } \\
\text { Ap stars } \\
\alpha \text { Cen }\end{array}$ \\
\hline $\begin{array}{l}\text { Multichannel/Multiline } \\
\text { a. Brown et al. } \\
1991 \\
\text { b. Libbrecht } \\
1988 \\
\text { c. Frandsen } \\
1987 \\
\text { d. Connes } \\
1985\end{array}$ & $\begin{array}{l}\text { HRS + fiber + scrambler } \\
+ \text { calibration lamp } \\
\text { HRS + fiber } \\
+ \text { calibration lamp } \\
\text { HRS + fiber } \\
\text { core/continuum ratio } \\
\text { HRS + fiber + scrambler } \\
+ \text { F-P + } 2 \text { lasers }\end{array}$ & $\begin{array}{l}\alpha \text { Cen, Procyon } \\
\gamma \text { Equ } \\
\beta \text { Hyi } \\
\text { none }\end{array}$ \\
\hline $\begin{array}{l}\text { Intermediate solution } \\
\text { a. Frandsen et al. } \\
1992\end{array}$ & $\begin{array}{l}\text { HHS interferometer } \\
+ \text { cross-disperser }\end{array}$ & none \\
\hline
\end{tabular}

Non multiplexing techniques

Although one can easily show that a proper S/N-ratio can not be obtained with any acceptable investment of telescope time by a filter type instrument, it still seems reasonable to try to detect oscillations in a few bright solar-type stars by this technique. References to such experiments can be found in Table II. The most convincing power spectrum is presented by Pottasch et al. (1992) and is 


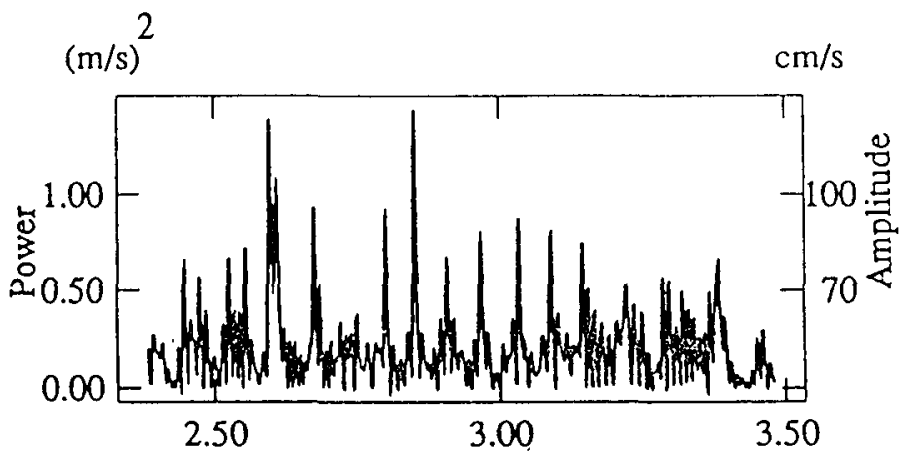

Frequency $(\mathrm{mHz})$

FIGURE II Power spectrum of $\alpha$ Cen A observed by Pottasch et al. 1992. Equally spaced peaks seems to be present as seen on the Sun

given here as Figure II. Still, one does not have a completely solid proof of the existence of $p$-modes in a star, but if the peaks identified by Pottasch et al. was detected in an independent experiment, this would probably be enough to convince most people about the reality of the modes.

Based on the results obtained so far, it is evident, that future work in this direction for solar type stars hold no promise. One must use more efficient instrumentation. Filter type instruments, although, represent an interesting solution to study slower, larger fluctuations in giants or radial velocity changes due to planets.

\section{Multiplexing techniques}

It is a straight forward idea that a high resolution spectrograph might be employed, provided it could be calibrated well enough. Simple as it sounds, in practice it has proven very difficult. Similar difficulties have been encountered in the search for planets around stars.

Nevertheless this is in the end the only efficient way to observe Doppler velocities and several groups are working on the perfection of the technique to have measurements of a quality close to the theoretical noise limit. Still much need to be done. The ultimate solution has been formulated by Connes (1985), but the design contain a number of technical innovations, that has to be made to work. Other less ambitious projects use fiber coupled spectrographs built for precise radial velocity measurements and include the MUSICOS project (Baudrand and Böhm, 1992), the AFOE (Noyes et al. 1992) and the VELUX spectrograph (Andersen et al. 1992).

Figure III, a modified version of a figure from Connes (1985), demonstrates that even with modest telescopes solar type $p$-modes can be detected in slow rotators with a modest effort given the right instrumentation. One can even go to rather faint magnitudes. More discussions about detection limits can be found in Appourchaux (1991) and Brown (1990b).

A drawback of spectrographs is that they have to be matched to the tele- 


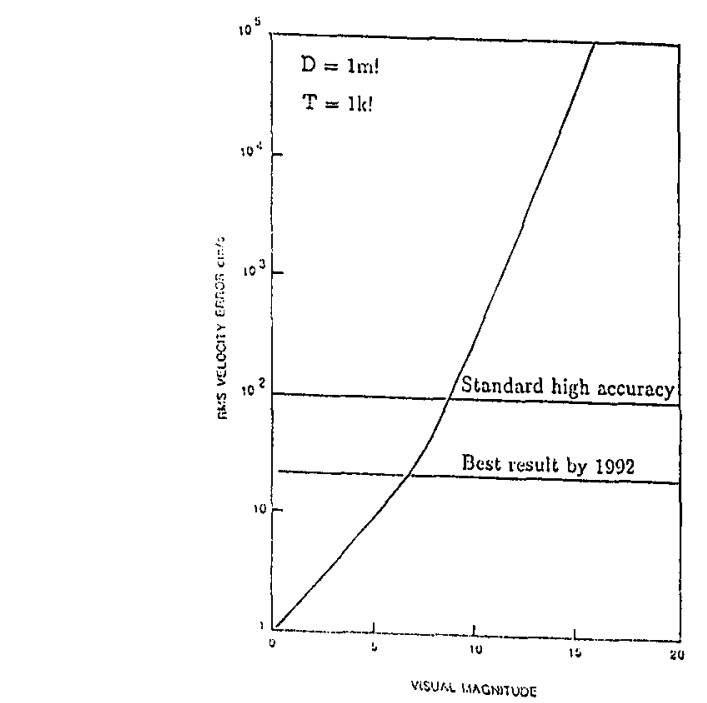

FIGURE III Observational limits

scopes. Also they tend to become large instruments, which are hard to make stable, even when used in a highly differential mode. It can be very difficult to locate the source of instrumental noise, which come from subtle mechanical and optical imperfections of the instrument.

An intermediate solution in terms of efficiency is a multiplexed interferometer as presented by Frandsen et al. (1992). This combines the stability of the filter techniques with some multiplex gain. It also provides a flexible instrument that can be used at many different telescopes without modifications. It involves some new technology, which yet have to be shown to work.

It can be stated that progress in this field of Doppler techniques is taking place, but slowly, and it will take some years before the technique will provide the very high quality data needed. In addition to the technical problems, multisite campaigns need to be set up to get nice, clean spectra, which poses an organizational challenge.

\section{INTENSITY STUDIES}

The intensity oscillation amplitudes for solar type stars are very small, only ppm's, whereas the other classes have amplitudes in the mmag range. To enable real seismology long observing periods are needed for all variables and the less interruptions the better.

As holes in the time series are difficult to avoid, even for large groups of telescopes like WET, very good $\mathrm{S} / \mathrm{N}$ data is required to enable a deconvolution of the window function.

The smallest detected amplitudes so far are of the order a few tenth of a mmag. Recently attempts to detect solar type oscillations in stars in M67 are 


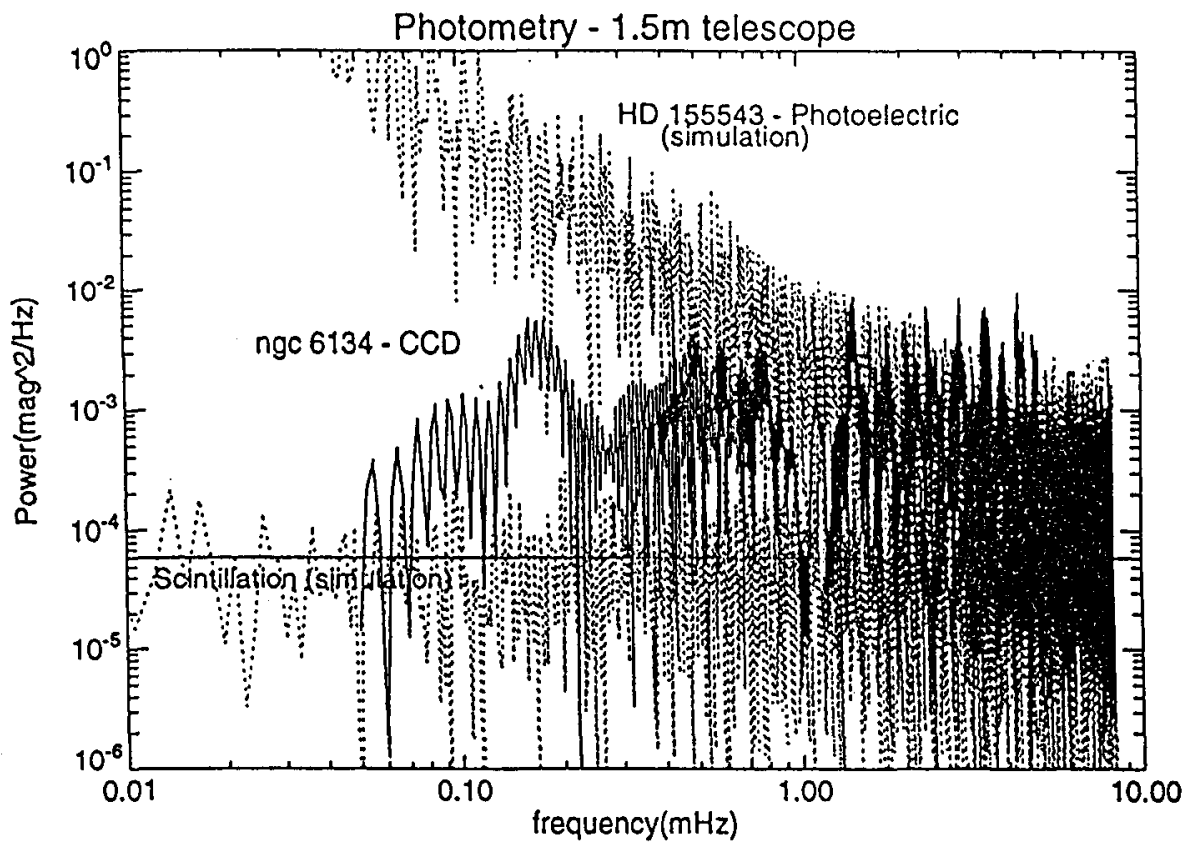

FIGURE IV A figure from Kjeldsen and Frandsen (1992). The curves have been scaled to correct for the difference in observing time. The photoelectric simulation matches the observed power spectrum as given by Belmonte et al. (1990) There is no big difference in the $3 \mathrm{mHz}$ range, but in the $\delta$-Scuti range $(0.1 \mathrm{mHz})$ the differences become very large between CCD and photoelectric photometry

expected to lead to an order of magnitude improvement in detection sensitivity (Gilliland et al. 1990).

The use of CCD detectors in high precision photometry has expanded the possibilities due to the stability of this detector type, and the option to make simultaneous, differential photometry for many objects. The limiting factor now is the available photons or the scintillation. For stars above the scintillation limit there is not much that can be done to improve $S / N$, because the noise decreases only slowly with the diameter of the telescope.

An investigation of photometry with CCD's by Kjeldsen and Frandsen (1992) demonstrate the superiority of CCD measurements especially at very small amplitudes and at low frequencies. Figure IV show actual data and simulated data where it is evident that especially the $1 / f$ noise is much smaller for CCD data than photoelectric data. At some observatories conditions are so stable that photoelectric work does not add any atmospheric noise (except scintillation) to the photon noise, but this is normally not the case. 
TABLE III Classes of variables

\begin{tabular}{|l|l|l|l|}
\hline Observatory & $\bar{D}$ & Period allocated & $\%$ contr. \\
\hline Kitt Peak & $4.0 \mathrm{~m}$ & 11-16 Jan & 23.9 \\
CFHT & $3.6 \mathrm{~m}$ & 12-16 Jan & 18.7 \\
AAT & $3.9 \mathrm{~m}$ & 14-17 Jan & 8.1 \\
Calar Alto & $3.6 \mathrm{~m}$ & 14-16 Jan & 4.9 \\
Nordic Opt. & $2.5 \mathrm{~m}$ & 12-17 Jan & 7.7 \\
Palomar & $5.0 \mathrm{~m}$ & 11-17 Jan & 36.7 \\
Kavalur & $2.3 \mathrm{~m}$ & xx-xx Jan & 0.0 \\
\hline
\end{tabular}

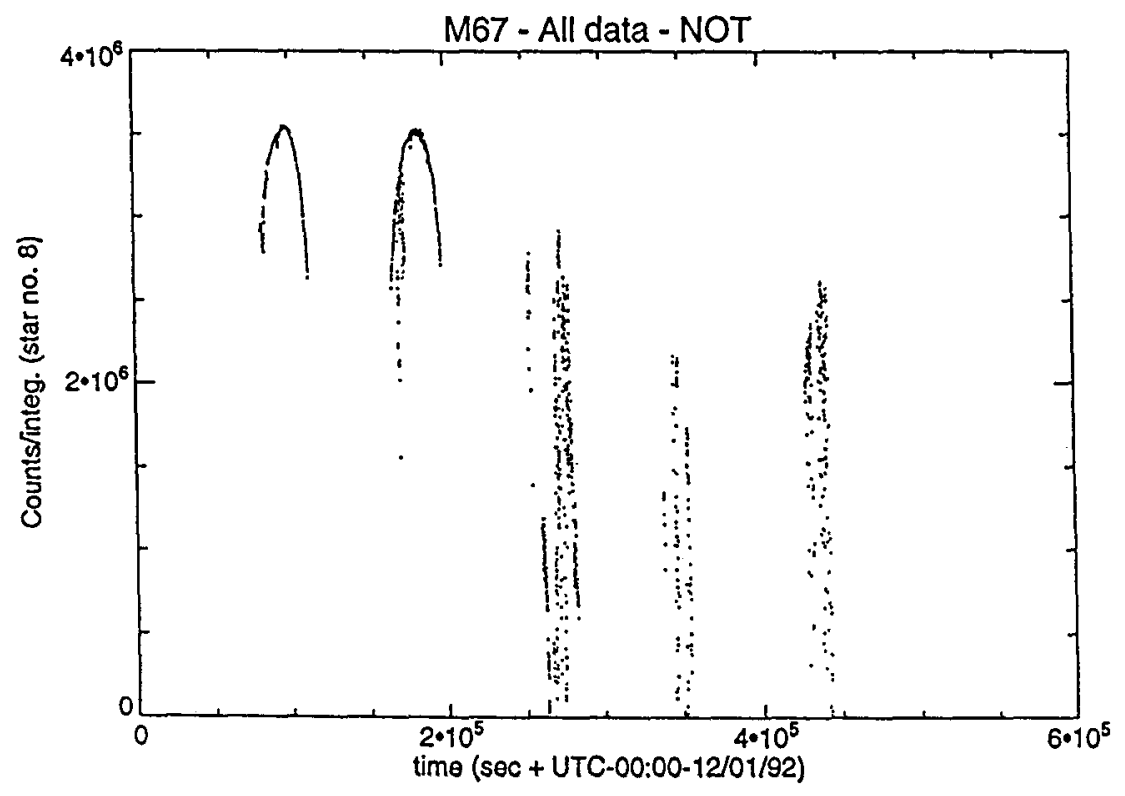

FIGURE V Record of the data observed by the NOT $2.5 \mathrm{~m}$ during the January 92 campaign. Fluxes are given for a particular star. 
Photometry from the ground

With large format CCD's the situation has improved for making high precision differential photometry: Using the formulas from Kjeldsen and Frandsen (1992), and choosing an effective filter width of $\Delta \lambda=100 \mathrm{~nm}$ and an integration time of $t=60 \mathrm{~s}$, we get for the magnitude $m$ for which scintillation and photon noise is equal the result

$$
m=5.1+5 / 3 \log (D)
$$

where the diameter of the telescope $D$ is in $\mathrm{cm}$. For stars brighter than this scintillation dominates. Scintillation has the fairly slow dependence on diameter $\left(D^{-2 / 3}\right)$, and it is advantageous to use smaller telescopes and longer observing periods instead of applying for costly time on large telescopes.

Another advantage of using a small telescope is that the field of view increases. If we look at the number of stars in a CCD frame of $30 \times 30 \mathrm{~mm}^{2}$, assuming the density of stars to be uniform in space and 10000 stars brighter than $m=6$, we get for the number of objects in the field of view bright enough to be scintillation dominated

$$
n_{o b j}=9.18 \times 10^{2}(d / f)^{2} D^{-1}
$$

where $d$ is the detector size in $\mathrm{cm}$ and the $f$ the f-ratio of the telescope, typically $6<f<15$. For a small telescope like the $50 \mathrm{~cm}$ telescopes at La Silla with $f=13.6$ and a detector with $d=3 \mathrm{~cm}$ we find in the field an average of 0.9 stars per frame. The example is for a fairly slow telescope. A modern telescope with $f=6$ would give several stars per frame. The increase of detectors from around $1 \mathrm{~cm}^{2}$ to of the order $10 \mathrm{~cm}^{2}$ is seen to be quite important. For a given f-ratio the chances of finding suitable comparison stars decrease linearly with telescope diameter.

For a given star the largest telescope might not be the optimal instrument if comparison stars are not available in the field. Generally, for a small telescope, we should be able to find bright comparison stars, in the sense that they contribute no more to the noise than the program star. Of course, near the galactic plane, the situation is better than for the average sky, and near the poles worse.

\section{Large telescope campaigns}

It is interesting to explore how far one can come with photometric techniques. The most ambitious project so far is the latest campaign (Jan. 92) on M67 organized by Gilliland and Brown (1991), where a number of large telescopes was pointed towards a small group of stars for 5-6 nights. The data accumulated is given in the final column of Table III as the weighted percentage of the data from each site. The total number of useful nights was around 20.

Among the participating telescopes was the Nordic Telescope at La Palma, and Figure $V$ shows the raw magnitudes for one of the stars from 5 nights of data.

Based on the reduction of data from Kitt Peak the extrapolated noise in the interesting frequency range is $7 \mu \mathrm{mag}$, very close to the level that was estimated as the value for a successful campaign. The result still depends on whether merging the data can be done without losses. It is also assuming that the data 


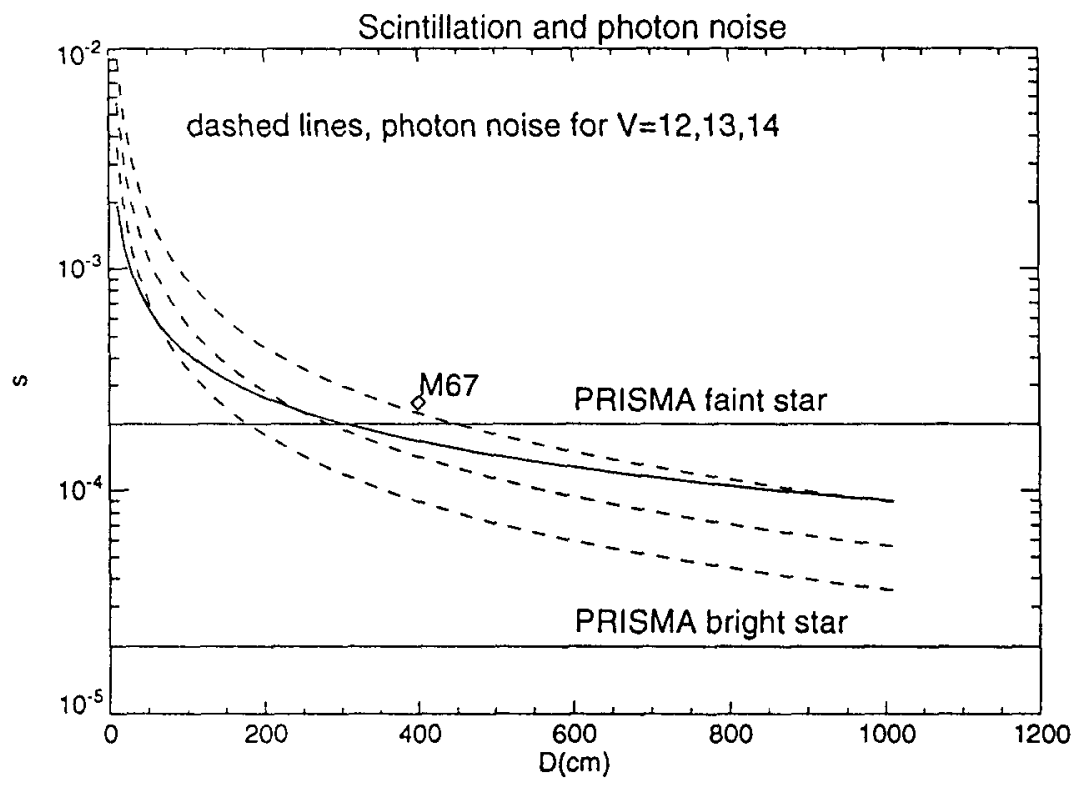

FIGURE VI The noise per frame $(t=60 s)$ as function of the diameter $D$ is plotted for scintillation noise (full drawn) and photon noise (dashed). The preliminary result for the Kitt Peak $4 \mathrm{~m}$ is indicated by the symbol. The horizontal lines present the noise for the brightest and the faintest star observed with the Large Photometer on PRISMA, where ppm accuracy will be reached.

from other telescopes can be used as efficiently as the Kitt Peak data. Data from several sites have been reduced and looked at and no serious problems discovered so far.

The results obtained can be compared with the curves in Figure VI, which at the same time illustrate how difficult it will be to do much better. In this figure the noise level due to scintillation and counting statistics is plotted against aperture of a telescope. Magnitudes typical for the selected M67 stars are plotted as dashed lines.

\section{Results from long time series}

As modes often occur in $\delta$-Scuti stars or white dwarfs with very small frequency differences, like a few $\mu \mathrm{Hz}$, the modes can only be resolved when long time series are obtained, where long is of the order a week for white dwarfs and several weeks for $\delta$-Scutis.

The gain in quality of the power spectra measured is evident when one looks at the WET results for white dwarfs (Winget et al. 1990), where hundred of modes have been identified. Piece of a spectrum was shown as Figure I. 


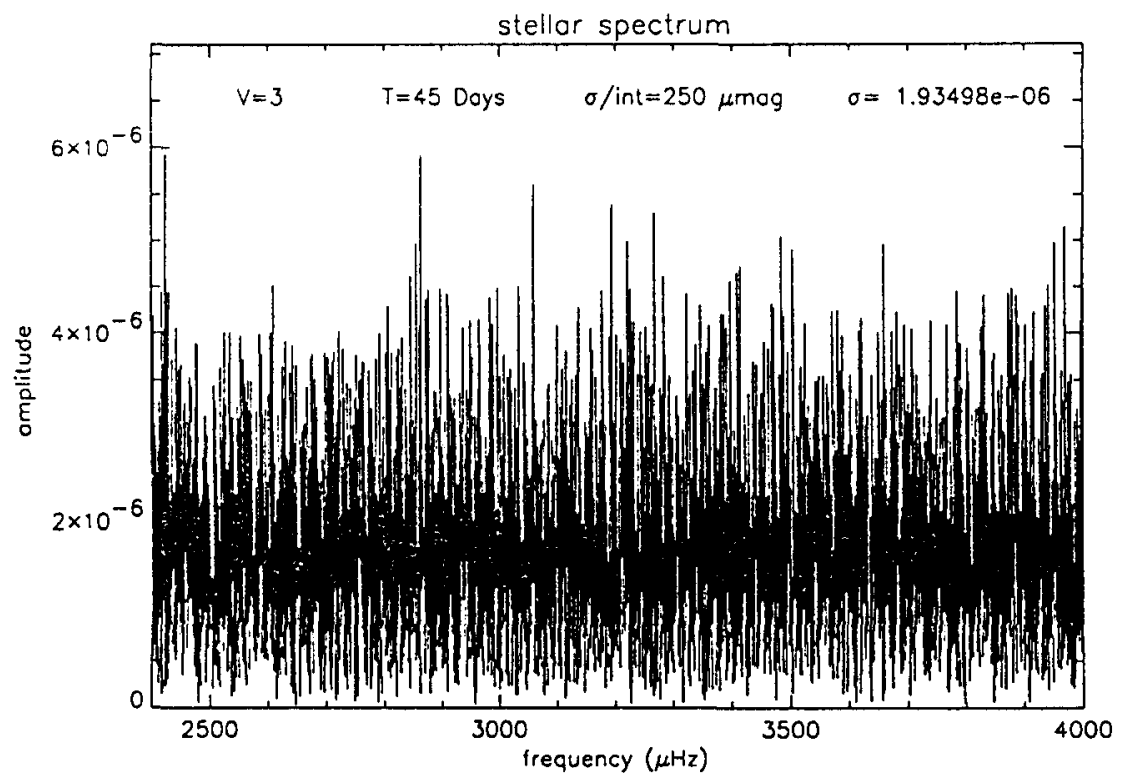

FIGURE VII A simulated amplitude spectrum obtained by a network of $4 \mathrm{~m}$ telescopes. Integration time 60s. The noise level per integration is indicated as well as the r.m.s. noise in the spectrum. A solar spectrum is in there, but the noise dominates to the extent, that no signal is seen at all.

\section{BEYOND THE ATMOSPHERE}

Even with a network of large telescopes intensity fluctuations due to $p$-modes in stellar analogs to the Sun can not be detected due to the scintillation in the atmosphere. The best way to illustrate this is by showing a simulation (Figure VII) of 45 days of data as observed by a network of $4 \mathrm{~m}$ class telescopes using a window function similar to what was obtained during the aforementioned M67 campaign. The noise per measurement is the typical value for a 60 s exposure obtained at the Kitt Peak 4m during the M67 campaign. Hidden in the noise is a simulated solar power spectrum, which clearly can not be detected.

In contrast to this an example of a simulation of a spectrum obtained with the large photometer on the PRISMA (report 1991) payload is shown as Figure VIII. It is evident, that the advantage of going to space is enormous. The same improvement in quality will be the case for all stars bright enough to be within the range of the PRISMA instruments. This selection of stars is very large as the faint limit for ppm noise level is 8th magnitude. Even at 5 magnitudes below this level, the noise level is still very small and will allow extremely interesting data to be collected for the higher amplitude variables.

Seismology as used to explore the Sun can only be applied to stars in a 


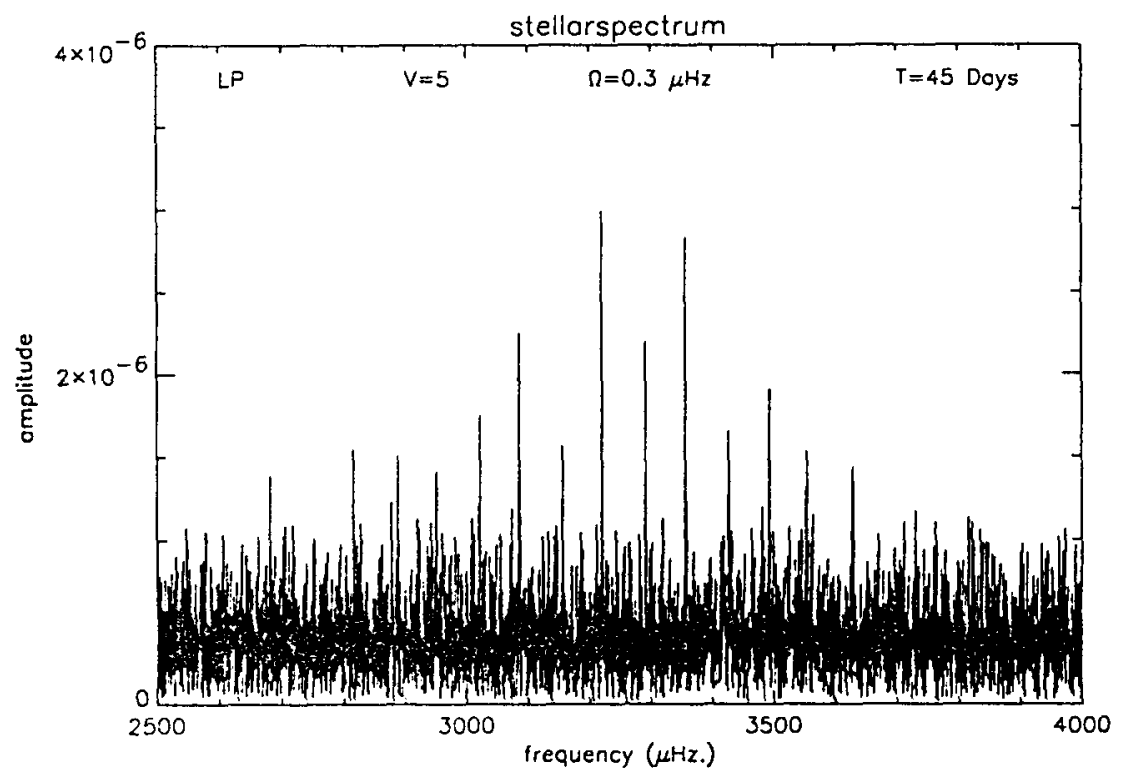

FIGURE VIII A simulated amplitude spectrum of the Sun at $V=5$ as observed by the Large Photometer on PRISMA. Indicated is the rotational rate $\Omega$ and the length of the observing period $T$. The r.m.s. noise level is $\sigma=3.1 \times 10^{-7}$. 
wider context by observing from space with a reduced noise level and continuous viewing exceeding one month. Only then can we measure frequencies precisely and study the separation between close frequencies, the shape of the peaks and the splitting due to rotation in slowly rotating stars.

But among all the interesting objects only a fraction can be among the targets of one space mission. The observations from space must be limited to a sample of objects, which are of particular interest in astrophysics. Everything should be done to improve ground based techniques to supplement and complement space observations, and to avoid more observations from space than absolutely necessary due to the high cost and long planning involved.

\section{REFERENCES}

Andersen, T., Dybdal, S., Frandsen, S. and Reiz, A. 1992, The NOT Newsletter 6 (VELUX)

Appourchaux, T. 1991, $A \mathscr{E} A$ 222, 361

Baade, D. 1991, in Rev. in Mod. Astr. 4, Springer

Baudrand, J. and Böhm, T. 1992, $A B A A$ accepted (MUSICOS)

Belmonte, J.A., Peres Hernández, F. and Roca Cortes, T. 1990, A\&A 331, 383

Brown, T.M. 1990a, The Sun and Asteroseismology, preprint

Brown, T.M. 1990b, in CCDs in Astronomy, Tuscon Arizona, ASP Conf. Ser. 8,335

Brown, T.M., Gilliland, R.L., Noyes, R.W. \& Ramsey, L.W. 1991, ApJ 368, 599

Connes, P. 1985, Astrophys. Sp. Sci. 110, 211

Frandsen, S. 1987, A\&A 181, 289

Frandsen, S., Douglas, N., Butcher, H. 1992, $A \& A$, submitted

Gilliland, R., Brown, T.M., Duncan, D.K., Suntzeff, N.B., Lockwood, G.W., Thompson, D.T., Schild, R.E., Jeffrey, W.A. and Penprase, B.E. 1991, AJ 101, 541

Gilliland, R. and Brown, T.M. 1991, Observing guide: M67 Stellar Oscillations Network Campagin, Private communication

Innis, J.L., Isaak, G.R., Speake, C.C., Brazier, R.I. \& Williams, H.K. 1990 preprint

Kjeldsen, H. and Frandsen, S. 1992 PASP, accepted

Libbrecht, K.G. 1988, "The Impact of very high S/N Spectroscopy in Stellar Physics", IAU Symp. 132, eds. Cayrel de Strobel, G. \& Spite, M., 83

Mantegazza, L., Poretti,E, Antonello, E., Riboni, E. and Zerbi, F. 1992, These Proceedings

Michel, E. and Baglin, A. 1991, Adv. Space Res. 11, 70

Nather, R.E, Winget, D.E, Clemens, J.C., Hansen, C.J. and Hine, B.P. 1990, ApJ 361, 309 (WET) 
Noyes, R.W, Brown, T.M., Horner, S., Korzennik, S. and Nisenson, P. 1992, These Proceedings (AFOE)

Pottasch, E.M., Butcher H.R. \& van Hoesel, F.H.J. 1992, $A \& A$, accepted

PRISMA, Report on the Assessment Study, January 1991, ESA SCI(91) 5

Reay, N.K., Ring, J., Pietraszewski, K.A.R.B., Bell, C.R. \& Leeper, M. 1986, SPIE Vol 627 Instrumentation in Astronomy VI, 27

Schmider, F.-X., Fossat, E., Grec, G. \& Gelly, B. 1988, Proc. Symp. Seismology of the Sun and the Sun-like Stars, Tenerife, Spain, ESA SP 286, 605

Schmider, F.-X. 1989, Thesis, Universite de Paris VII

Winget, D.E. and the WET consortium (27 authors) 1990, ApJ 357, 630 